\title{
Efecto de temperaturas que simulan incendios sobre la germinación de semillas de un bosque seco tropical
}

\section{Effect of temperatures that simulate fire on seed germination in a tropical diry forest}

\author{
Juan Carlos Cárdenas-Salgado ${ }^{10}$ y Camila Pizano ${ }^{\oplus}$
}

Cárdenas-Salgado, J.C. y Pizano, C. (2019). Efecto de temperaturas que simulan incendios sobre la germinación de semillas de un bosque seco tropical. Colombia Forestal, 22(2), 55-66.

Recepción: 5 de marzo de 2019

\section{Resumen}

El fuego es una perturbación recurrente pero poco estudiada en los bosques secos tropicales, en los que las semillas son la principal fuente de regeneración. En este estudio se determinó la tasa germinativa de semillas de ocho árboles del bosque seco colombiano expuestas a temperaturas elevadas que simulaban fuegos de baja $\left(100^{\circ} \mathrm{C}\right)$ y alta incidencia $\left(200^{\circ} \mathrm{C}\right)$ con diferentes tiempos de exposición (5 y 15 minutos). La temperatura fue el factor que mejor explicó la germinación de las semillas, mientras que el tiempo no tuvo un efecto significativo. Siete especies obtuvieron niveles de germinación similares bajo el tratamiento de $100^{\circ} \mathrm{C}$ y en el control, mientras que la germinación para el tratamiento de $200^{\circ} \mathrm{C}$ fue casi nula. Los resultados sugieren que las semillas de algunas especies de bosque seco presentan tolerancia a los incendios moderados, sin embargo, la mayoría de semillas perecerían bajo fuegos de alta incidencia.

Palabras clave: bosque seco tropical, incendios forestales, resiliencia, semillas, tasa de germinación.
Aprobación: 6 de mayo de 2019

\begin{abstract}
Fire is a recurring, but poorly studied disturbance in tropical dry forests, where seeds are the main source of regeneration. In this study, we measured seed germination of eight Colombian dry forest tree species under elevated temperature treatments that simulated fires of low $\left(100^{\circ} \mathrm{C}\right)$ and high intensity $\left(200^{\circ} \mathrm{C}\right)$ with different exposure times (5 and 15 minutes). We found that the factor that best explained seed germination was temperature, while time was not significant. Seven species germinated similarly under the treatment of $100^{\circ} \mathrm{C}$ and under the control, while seed germination under $200^{\circ} \mathrm{C}$ was almost null. These results suggest that the seeds of some dry forest species have tolerance to moderate fires, however, most seeds would perish under high intensity of fires.
\end{abstract}

Key words: tropical dry forest, fire, resilience, seed germination rate.

Universidad Icesi, Departamento de Ciencias Biológicas. Cali, Valle del Cauca, Colombia. juan.cardenas3@correo.icesi.edu.co. Autor para correspondencia.

Universidad Icesi, Departamento de Ciencias Biológicas. Cali, Valle del Cauca, Colombia. cpizano@icesi.edu.co 


\section{INTRODUCCIÓN}

El bosque seco tropical (BST) es uno de los ecosistemas con mayor trasformación debido a una larga historia de ocupación por sus suelos parcialmente fértiles y condiciones climáticas favorables (Pizano et al., 2016). En Colombia queda el $8 \%$ (cerca de 705000 ha) de las 9000000 ha que cubría originalmente el bosque seco en el territorio nacional y los bosques que quedan están altamente fragmentados (García, Corzo, Isaacs y Etter, 2014; Pizano et al., 2016; González-M et al., 2018), por lo cual el BST es uno de los ecosistemas más amenazados en el país. Más aún, en el país el $65 \%$ de las áreas que originalmente eran bosques secos y han sido transformadas, en la actualidad presentan un escenario de desertificación, lo cual indica el alto deterioro de este ecosistema (García et al., 2014; Rodríguez et al., 2016). Sin embargo, varios estudios recientes han encontrado que los remanentes de bosque seco todavía alojan un alto número de especies de plantas endémicas (Dryflor, 2016; González-M et al., 2017) y, por lo tanto, tienen un alto valor de conservación.

Las presiones que más han afectado históricamente al bosque seco incluyen el cambio climático, la fragmentación, la expansión de la agricultura y la ganadería y el fuego (Janzen, 1988; Pizano et al., 2016; González-M. et al., 2018). Este último se ha reconocido como una de las perturbaciones más recurrentes a nivel antrópico y natural en el BST, y su frecuencia e intensidad dependen de la estructura de la vegetación, el clima y la humedad, entre otros (Middleton, Sanchez-Rojas, Suedmeyer y Michels, 1997; Otterstrom, Schwartz y Velázquez-Rocha, 2006). De hecho, la mayoría de los incendios antrópicos en los bosques secos se debe a la presencia de pastos y matorrales invasores dentro del bosque, los cuales no solo son más inflamables, si no también más resilientes luego de un evento de fuego (D'Antonio y Vitousek, 1992; Monmany, Gould, Andrade-Núñez, González y Quiñones, 2017). Tanto gramíneas como matorrales han sido introducidos en las matrices de ganadería y la agricultura que rodean al bosque seco (González-M et al., 2018), lo cual ha generado el desplazamiento de especies nativas dentro del bosque (D'Antonio y Vitousek, 1992; Veldman, Mostacedo, Peña-Claros y Putz, 2009; Labbé, 2013). En particular, las gramíneas invasoras tienen mecanismos de reclutamiento rápido y resistencia post-incendio, por lo que el fuego fomenta su esparcimiento a través del bosque ( $\mathrm{D}^{\prime} \mathrm{An}$ tonio y Vitousek, 1992; Otterstrom et al., 2006; Labbé, 2013) y permite un aumento en la biomasa combustible (Veldman et al., 2009; Veldman y Putz, 2011). De esta manera, cuantos más pastos invaden el bosque, mayor es la probabilidad de incendios; y, a mayor número de incendios, mayor es el área del bosque colonizada por los pastos (Veldman et al., 2009; Veldman y Putz, 2011). Por el otro lado, una alta biomasa acumulada de pastos provoca incendios de mayor incidencia que pueden reducir la germinación de las semillas del bosque nativo, conduciendo a la degradación de estos ecosistemas (Otterstrom et al., 2006; Cordell et al., 2008). Efectivamente, en la mayoría de bosques secos del mundo se han registrado un incremento en la abundancia de pastos exóticos que invaden los claros, ligado a un aumento en la frecuencia, intensidad y área de los incendios en los bosques (D'Antonio y Vitousek, 1992; Veldman et al., 2009; Veldman y Putz 2010).

Sin embargo, el fuego también puede beneficiar a algunas especies de plantas del bosque seco. Por ejemplo, algunos estudios como el de Otterstrom et al. (2006) demostraron que los incendios experimentales aumentan la densidad de plántulas en los bosques secos de Nicaragua, indicando que algunas especies son tolerantes al fuego y que poseen estrategias adaptativas para la recolonización de áreas perturbadas. En contraste, otros estudios han demostrado que son pocas las plántulas de especies que sobreviven al fuego, como lo demostraron Dokrak, Utis, Hiroshi y Nakashizuka (2004) en los bosques secos de Tailandia. Con todo, son varios los estudios que han reportado estrategias ecológicas en respuesta al fuego, 
como la capacidad de rebrotar en el estadio de plántula gracias a grandes reservas de nutrientes en raíces engrosadas en los bosques secos (Bond y Parr, 2010; Bhadouria, Singh, Srivastava y Raghubanshi-Singh, 2016). Por el otro lado, algunos estudios reportan que las semillas de bosques secos, donde el fuego es recurrente, tienen integumentos gruesos que las protegen del fuego, o pueden escapar al fuego durante la época de lluvias cuando los incendios son poco probables (Khurana y Singh, 2001). Sin embargo, es poca la información que se tiene sobre cómo se ven afectadas las semillas de diferentes especies de bosque seco a incendios de diferentes intensidades. En particular, se desconoce cuál puede ser el impacto de los incendios de alta intensidad, los cuales son cada vez más frecuentes por la acumulación de biomasa de pastos exóticos en el BST, sobre la germinación de las semillas. En esta investigación se expusieron semillas de 8 especies de árboles comunes del bosque seco del Valle del Cauca a altas temperaturas que simulan fuego de baja y alta incidencia. Nuestra predicción era que las especies representativas de semillas de BST como Ceiba pentandra y Erythrina poeppigiana, (las cuales se han reportado como resistentes al fuego) (Khurana y Sigh, 2001), germinarían bajo los tratamientos de alta temperatura, mientras que las demás especies, solo germinarían bajo el control.

\section{MATERIALES Y MÉTODOS}

\section{Procedencia de las semillas}

Dado que el objetivo del estudio era trabajar con especies de árboles representativas del bosque seco del Valle del Cauca, se escogieron ocho especies que han sido reportadas como abundantes en el Parque Natural Regional (PNR) El Vínculo, uno de los bosques secos mejor estudiados en la región. Las semillas fueron suministradas por Camposeeds (figura 1). El PNR EI Vínculo está ubicado en el piedemonte de la cordillera Central ( $3^{\circ} 50^{\prime}$ $23^{\prime \prime}$ latitud N; $76^{\circ} 18^{\prime} 07^{\prime \prime}$ longitud W) a $3 \mathrm{~km}$ al sur del municipio Buga, y su altitud varía entre los 977 y los 1150 m (Torres et al., 2012). Este parque contiene el fragmento más grande de BST del valle geográfico del río Cauca (Torres et al., 2012), con una superficie de 98 ha y 58 ha de bosque seco intangible. La temperatura media anual es de $24^{\circ} \mathrm{C}$ y cuenta con una precipitación promedio anual de $1379 \mathrm{~mm}$. El clima en esta zona tiene un comportamiento bimodal con dos periodos secos entre
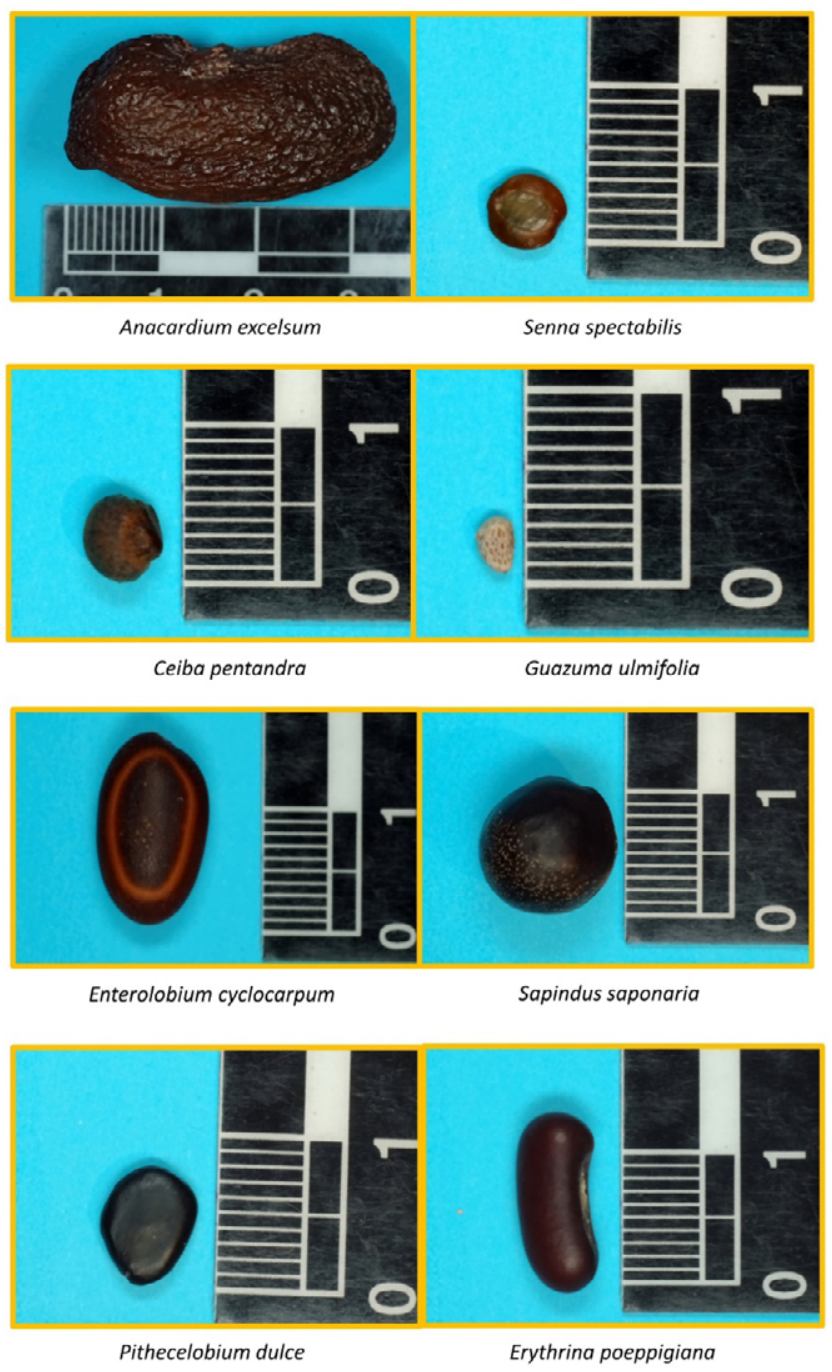

Figura 1. Semillas de especies de árboles abundantes en el Parque Natural Regional El Vínculo (Buga, Valle del Cauca) utilizadas en el experimento. 
enero a abril y de julio a agosto, y dos periodos de Iluvia, entre marzo a junio y de septiembre a diciembre. Estas características climáticas ubican al PNR El Vínculo en la zona de vida de BST según la escala de Holdridge (1967).

El peso seco de cada especie de semilla se estimó de 20 semillas por especie, las cuales se secaron en un horno a $60^{\circ} \mathrm{C}$ durante dos días y luego fueron pesadas en una balanza (tabla 1). También se le asignó el grupo ecológico a cada especie según Vargas (2012) y Henao, Torres, Tafur y Guevara (2018). Esto con la finalidad de determinar qué características podrían tener las semillas resistentes a las altas temperaturas, ya que según Romero-Saritama y Pérez-Ruiz (2016), las semillas grandes y pesadas (alrededor de $3 \mathrm{~mm}$ ) tienen gran cantidad de ácidos grasos y altos contenidos de humedad, por lo que suelen ser menos resistentes a altas temperaturas. Finalmente, las semillas de cada especie fueron fotografiadas con una Cámara Nikon D70S y un lente $55 \mathrm{~mm}$ Micro-Nikkor.

Tabla 1. Peso seco promedio ( \pm desviación estándar) y grupo ecológico de las 8 especies de semillas utilizadas en el experimento.

\begin{tabular}{llc}
\hline \multicolumn{1}{c}{$\begin{array}{c}\text { Especies de } \\
\text { semillas }\end{array}$} & \multicolumn{1}{c}{$\begin{array}{c}\text { Grupo } \\
\text { ecológico }\end{array}$} & Peso seco (g) \\
\hline $\begin{array}{l}\text { Enterolobium } \\
\text { cyclocarpum }\end{array}$ & Pionera & $0.633 \pm 0.07$ \\
$\begin{array}{l}\text { Guazuma ulmifolia } \\
\text { Sapindus saponaria }\end{array}$ & Pionera & Pionera Intermedia \\
$\begin{array}{l}\text { Pithecellobium } \\
\text { dulce }\end{array}$ & Pionera Intermedia & $0.698 \pm 0.096$ \\
$\begin{array}{l}\text { Ceiba pentandra } \\
\text { Senna spectabilis }\end{array}$ & Pionera Intermedia & $0.048 \pm 0.0018$ \\
$\begin{array}{l}\text { Erythrina } \\
\text { poeppigiana }\end{array}$ & Pionera Intermedia & $0.025 \pm 0.003$ \\
$\begin{array}{l}\text { Anacardium } \\
\text { excelsum }\end{array}$ & Pionera Intermedia & $0.170 \pm 0.04$ \\
\hline
\end{tabular}

\section{Experimento de invernadero}

Las semillas (20 por especie) se sometieron a diferentes temperaturas que pretendían simular las que experimentan las semillas a una profundidad de $3 \mathrm{~cm}$ en el suelo en incendios de alta y baja intensidad en un bosque seco (Kennard y Gholz, 2001). El experimento consistió de tres tratamientos: control (sin calentar), $100^{\circ} \mathrm{C}$ (simulando fuegos de baja intensidad), y $200^{\circ} \mathrm{C}$ (simulando fuego de alta intensidad). Las semillas bajo tratamientos de calentamiento se colocaron en bandejas de aluminio de $15.2 \mathrm{~cm}$ de largo x $12.7 \mathrm{~cm}$ de ancho x $5.1 \mathrm{~cm}$ de alto marca Darnel ${ }^{\circledR}$ con una mezcla de suelo y cascarilla de arroz, de tal manera que las semillas quedaban sobre la superficie del suelo en cada bandeja. Posteriormente, se expusieron a altas temperaturas en una cámara de calor y secado Binder modelo ED 53 (Binder $\mathrm{GmbH}$ ) durante diferentes tiempos: 15 min y 5 min, teniendo en cuenta los resultados encontrados por Carrington (2010) y Wanthongchai, Goldammer, y Bauhus (2011) sobre la duración de incendios forestales. Tras los tratamientos respectivos, las semillas fueron sembradas en germinadores individuales en el invernadero de la Universidad Icesi con una mezcla de suelo y cascarilla de arroz con el objetivo de mejorar el drenaje. Este fue previamente autoclavado (ciclo sólido: $120^{\circ} \mathrm{C}$ y 15 psi durante 90 minutos) para eliminar semillas o plántulas indeseadas. La germinación (emergencia del hipocotilo por encima de la superficie del suelo) se monitoreó semanalmente durante 7 semanas.

\section{Análisis estadísticos}

Los resultados de germinación de semillas (proporción de germinación) se analizaron con el programa R (versión 3.4.0), la plataforma Rstudio (versión 1.0.143) y el paquete ggplot2 (Wickham, 2005). La germinación total para cada especie y cada tratamiento se estimó con un modelo lineal generalizado (GLM) asumiendo una distribución binomial de los datos y utilizando el criterio de información de Akaike (AIC). Bajo una distribución binomial la variable dependiente oscilaba entre 0 y 1 , es decir, germinación o no germinación para cada una de las especies bajo cada tratamiento (Minitab18, 2017), y las variables independientes se definieron 
como la temperatura y el tiempo de calentamiento. Los parámetros utilizados para crear los modelos fueron los siguientes:

\section{Modelos lineales generalizados: familia de} distribución binomial

- Modelo 1: Variables: Tiempo, Temperatura e interacción Tiempo - Temperatura.

- Modelo 2: Variables: Tiempo, Temperatura.

- Modelo 3: Variable: Tiempo.

- Modelo 4: Variable: Temperatura.

- Modelo nulo: No tiene en cuenta ningún parámetro.

Para definir la plausibilidad de las variables evaluadas en el experimento se utilizó el criterio de información de Akaike (AIC) (Akaike, 1974), el cual evalúa y compara el poder de predicción de un conjunto de modelos estadísticos entre sí, estimando el que mejor se ajuste a los datos (Burnham y Anderson, 2003). Este criterio se utiliza para definir cuál de los modelos tiene el mejor ajuste con el valor de dAIC, es decir, la diferencia entre el valor de AIC de un modelo y el valor más bajo de todos los AIC. Por lo tanto, en la escala relativa de plausibilidad, los modelos con valores de dAIC en un intervalo entre 0 y 4 (rango que reduce el error tipo I), son los modelos con máxima verosimilitud para los factores que se escogieron. Considerando que los dAIC de la mayoría de los modelos se encontraban dentro de este rango (anexo 1), se utilizó el principio de parsimonia para seleccionar el modelo con menos factores que pudiera explicar la variación de los datos (Burnham y Anderson, 2003). Finalmente, se comparó la proporción de germinación para cada especie utilizando un test de proporciones de 2-muestras, el cual se concentra en establecer las diferencias que hay entre dos muestras (en este caso, la diferencia de germinación entre tratamientos). De esta manera, se evaluó si existían diferencias significativas en la germinación de semillas entre los tratamientos experimentales de control, T100, T200 utilizando un alfa $(\alpha)$ de 0.05 .

\section{RESULTADOS}

\section{Germinación de semillas bajo diferentes tratamientos}

De las ocho especies de árboles incluidas en el experimento, siete germinaron bajo el control, mientras que de Anacardium excelsum solo emergió una semilla; sin embargo, su hipocotilo no se desarrolló. Dado que las semillas fueron obtenidas de un proveedor, es muy probable que las semillas de esta especie estuvieran inviables por la posible pérdida de ácidos grasos, los cuales tienden a perderse debido a un mal almacenamiento (temperatura o humedad inadecuadas) o por envejecimiento (más de dos meses a $6^{\circ} \mathrm{C}$, es un almacenamiento inadecuado para esta especie) (Khurana y Singh, 2001; Morales, 2016). Como consecuencia, los resultados para esta especie no se incluyeron en la discusión.

Para las especies Enterolobium cyclocarpum, Guazuma ulmifolia, Sapindus saponaria, Erythryna poeppigiana y Anacardium excelsum el modelo que mejor explicó la variación de los datos fue el modelo nulo con un dAIC de $(2.4,2.4,3.5,3.8,0)$ respectivamente (anexo 1). Es decir, que como en general estas especies presentaron una baja tasa de germinación, ninguno de los factores explicó la variación de los datos. Por otro lado, para las especies Pithecellobium dulce, Ceiba pentandra, Senna spectabilis, el modelo M4 fue el más viable, con un valor de dAIC de 0 (anexo 1). Dicho de otra forma, que la temperatura (variable más parsimoniosa en el intervalo dAIC de 0-4) fue el factor que mejor explicó la variación para la probabilidad de germinación de estas especies, las cuales obtuvieron una germinación de más del $20 \%$ (Senna spectabilis), $50 \%$ (Ceiba pentandra) y $75 \%$ (Pithecellobium dulce), tanto para el control como para el tratamiento de $100^{\circ} \mathrm{C}$. Dado que el factor tiempo no explicó la variabilidad de la germinación para ninguna especie, solo se compararon los resultados de germinación obtenidos para el control y los dos tratamientos de temperatura (T100 y T200). 
En general, la germinación de las semillas de siete de las ocho especies, así fuera baja, fue similar para el control y bajo la temperatura de $100^{\circ} \mathrm{C}$, lo que indica que la mayoría de las especies utilizadas demostró tolerancia a los tratamientos de baja incidencia. En contraste, la germinación de las ocho especies fue casi nula bajo el tratamiento de $200^{\circ} \mathrm{C}$ (a excepción de una semilla de Sapindus saponaria), lo cual sugiere que la mayoría de las especies no germinaría en presencia de incendios de alta intensidad.

\section{DISCUSIÓN}

El fuego se ha reconocido como una de las perturbaciones más recurrentes en los bosques secos dado que su frecuencia e intensidad pueden determinar la distribución y composición de estos bosques (Middleton, Sanchez-Rojas, Suedmeyer y Michels, 1997; Otterstrom, Schwartz y
Velázquez-Rocha, 2006). En particular, el fuego en los bosques secos puede ser de origen natural (Goldammer, 1993; Middleton et al., 1997; Khurana y Singh, 2001; Otterstrom et al., 2006) o antrópico (Goldammer, 1993; Khurana y Singh, 2001; Vieira y Scariot, 2006). Por lo anterior, varias especies son tolerantes a las altas temperaturas generadas por los incendios (Goldammer, 1993; Khurana y Singh, 2001). Sin embargo, esa tolerancia varía según la plasticidad adaptativa de las especies, la cual está ligada a su historia ecológica o al área geográfica en la que se encuentren (Khurana y Singh, 2001; Kennard y Gholz, 2001).

En contra de nuestra predicción de que solo dos especies de bosque seco (Ceiba pentandra y Erythrina poeppigiana) iban a germinar bajo tratamientos de calentamiento, en este estudio siete de ocho especies de semillas de árboles de bosque seco germinaron en el control y bajo temperaturas de $100^{\circ} \mathrm{C}$ (figura 2), la cuales simulaban fuegos de baja incidencia. Los análisis estadísticos
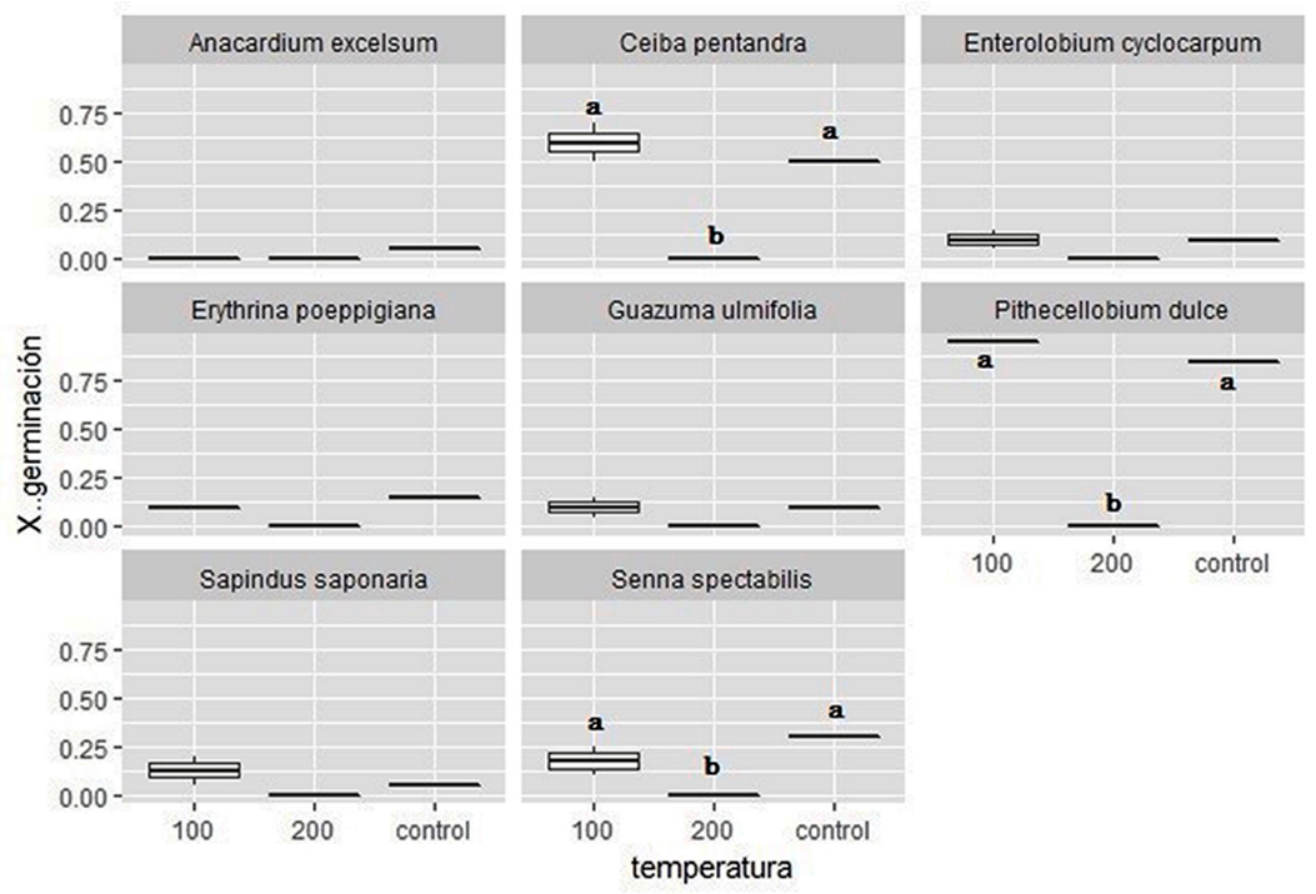

Figura 2. Proporción de germinación de cada una de las 8 especies de semillas de árboles abundantes en el Parque Natural Regional El Vínculo (Buga, Valle del Cauca). Las letras (a y b) indican diferencias significativas entre los tratamientos. 
demostraron que la temperatura fue el único factor que explicó la variación en la germinación de tres de estas ocho especies (Ceiba pentandra, Pithecellobium dulce y Senna spectabilis), la cual fue igualmente alta para el tratamiento de $100^{\circ} \mathrm{C}$ y el control, mientras que su germinación fue nula para el tratamiento de $200^{\circ} \mathrm{C}$ (figura 2). Esto sugiere que las semillas de estas especies son resistentes a incendios de baja incidencia (Kennard y Gholz, 2001) respectivos a disturbios naturales en los bosques secos tropicales (Goldammer, 1993; Middleton et al., 1997; Khurana y Singh, 2001; Otterstrom et al., 2006).

Cabe resaltar que estas tres especies son pioneras intermedias y tienen semillas que pesan menos de 1 gramo (tabla 1), lo cual, según Romero-Saritama y Pérez-Ruiz (2016) indica que tienen una tendencia ortodoxa. Es decir, que poseen bajos contenidos de humedad y una testa dura-impermeable, lo cual restringe la pérdida de humedad y, por consiguiente, son más resistentes a altas temperaturas (Gómez-González, Torres-Díaz, Bustos-Schindler y Gianoli, 2011).

Otras especies como Enterolobium cyclocarpum, Guazuma ulmifolia y Sapindus saponaria también tienen semillas pequeñas (menos de $1 \mathrm{~g}$ ) pero mostraron una baja germinación, probablemente debido a que estaban viejas y, por lo tanto, inviables. No obstante, los valores de germinación fueron similares para el control y en el tratamiento de $100^{\circ} \mathrm{C}$ al igual que las tres especies que sí respondieron a los tratamientos (Ceiba pentandra, Pithecellobium dulce y Senna spectabilis). Estos resultados pueden estar relacionados con el hecho de que cerca del $90 \%$ de las semillas de bosque seco son ortodoxas, lo cual puede estar asociado al hecho de que estos bosques presentan climas secos con altas temperaturas y poca humedad, los cuales pueden favorecer los incendios (Gómez-González et al., 2011; Romero-Saritama y Pérez-Ruiz, 2016). Es decir, que presiones selectivas como el fuego han condicionado la ecología de las especies del bosque seco y, por ende, a los rasgos morfológicos y fisiológicos de sus semillas (Gómez-González et al.,
2011). Por ejemplo, las altas temperaturas pueden escarificar la testa de algunas semillas, lo cual promueve su germinación. Esta escarificación puede ser similar a la escarificación química de la digestión animal. De hecho, Gómez-González, Cavieres y Pausas (2017) proponen que algunas leguminosas como Senna spectabilis y Pithecellobium dulce pueden ser tolerantes al fuego porque generalmente son dispersadas por animales, y la digestión intestinal es similar al choque térmico producido por el fuego. Por el otro lado, estudios como los de Gomez-Gonzalez et al. (2011), han demostrado que las semillas pequeñas y redondas tienden a ser más tolerantes a las altas temperaturas porque por su tamaño y forma, se les facilita enterrarse en el banco de semillas en el suelo, donde pueden evitar choques térmicos repentinos como los ocasionados por incendios superficiales (Bond, Honig y Maze, 1999; Hanley, Unna y Darvill, 2003).

En contraste con el tratamiento de $100^{\circ} \mathrm{C}$, solo una semilla de Sapindus saponaria germinó bajo el tratamiento de $200^{\circ} \mathrm{C}$ (figura 2), el cual simulaba fuegos de alta incidencia recurrentes en bosques secos con una alta abundancia de pastos y arbustos introducidos. Por lo que se puede suponer que temperaturas tan altas pueden provocar daños al embrión, tal y como lo demostraron Kennard y Gholz (2001) y Viera y Scariot (2006), quienes establecieron que los incendios de alta intensidad causan una disminución del $93 \%$ de la densidad de semillas y un $81 \%$ en la riqueza de semillas en el banco de semillas de BST. Por el otro lado, estudios recientes en los bosques secos del Valle del Cauca han demostrado que las especies herbáceas son abundantes en los bancos de semillas de estos bosques (Cárdenas, 2018; Erazo, 2016). Esto sugiere un proceso de sabanización del bosque seco en esta región (Janzen, 1988; D’ Antonio y Vitousek, 1992; Veldman y Putz, 2011), el cual puede ir ligado a una alta ocurrencia de incendios de alta incidencia que pueden afectar de manera negativa a las especies nativas de bosque seco (Veldman et al., 2009; Veldman y Putz, 2010; Veldman y Putz, 2011). Gómez-González et al. (2017) también 
mencionan que la gran cantidad de combustible generado por las especies de pastos inflamables produce fuegos intensos que amenazan seriamente los bancos de semillas donde aguardan las especies nativas para regenerar el área.

El caso de Sapindus saponaria es interesante porque esta especie empezó a germinar a partir de la cuarta semana, lo cual indica una germinación retrasada que puede estar relacionada con el hecho de que sus semillas tienen testa dura y, por ello, hidratación e intercambio de gases restringidos (Norden, Daws, Antoine, Gonzalez, Garwood y Chave, 2009). Estas características también podrían explicar por qué fue la única especie que mostró emergencia en el tratamiento de $200^{\circ} \mathrm{C}$, y, por lo tanto, sería la única especie que podría sobrevivir a eventos de fuego de alta incidencia. Sin embargo, estos resultados se deben interpretar con precaución por el reducido número de semillas utilizado en el experimento y las pocas semillas que germinaron. Por el otro lado, las semillas de Guazuma ulmifolia, Sapindus saponaria, Enterolobium cyclocarpum y Erytrhina poeppigiana tuvieron pocos individuos emergentes, y por ende no tuvieron una respuesta significativa a los tratamientos. Especulamos que estas semillas estaban inviables porque estaban envejecidas, o habían experimentado un mal almacenamiento con temperaturas o humedad inadecuados (Romero-Saritama y Pérez-Ruiz, 2016). Es necesario realizar nuevas investigaciones con un mayor número de especies de plantas de bosque seco y mayor replicación. Estos estudios serían pertinentes dada la necesidad de encontrar especies que sean resistentes a factores como el fuego para ser utilizadas en ejercicios de restauración de bosque seco (Otterstrom et al., 2006).

\section{CONCLUSIONES}

Dado que las semillas representan el potencial regenerativo de los bosques secos, estudiar su ecología es fundamental para entender los procesos como el reclutamiento de plantas y la sucesión, así como para asegurar el éxito de diferentes esfuerzos de restauración (Khurana y Singh, 2001). En este estudio siete de ocho especies germinaron bajo temperaturas de $100^{\circ} \mathrm{C}$, lo cual sugiere que presentan tolerancia hacia los incendios de baja intensidad, relacionados con incendios naturales. En contraste, solo una semilla de una especie (Sapindus saponaria) germinó bajo el tratamiento de $200^{\circ} \mathrm{C}$, indicando que las semillas de la mayoría de especies de bosque seco no sobrevivirían a incendios de alta incidencia típicos de bosques intervenidos con alta frecuencia de especies inflamables como los pastos. Es decir, que de seguir la presente tendencia de degradación del bosque seco y el incremento de las presiones antropogénicas como el fuego sobre estos bosques (González-M et al., 2018), será cada vez más difícil su regeneración a partir de semillas. Como consecuencia, es necesario realizar nuevos estudios con un mayor número de especies y mayor replicación con el objetivo de encontrar especies que sean resistentes a factores de presión como el fuego para ser utilizadas en ejercicios de restauración. En particular, dado que la composición del bosque seco varía en la región (González-M et al., 2018), se deben investigar las especies más representativas de cada bosque seco para asegurar procesos de restauración más exitosos.

\section{AGRADECIMIENTOS}

Agradecemos a la Universidad Icesi por proporcionar los recursos económicos para llevar a cabo este estudio. A los profesores e investigadores del Herbario de la Universidad del Valle por su ayuda en la identificación de las semillas, y al Inciva por otorgarnos los permisos para realizar la recolección de muestras de semillas en el PNR EI Vínculo.

\section{CONFLICTO DE INTERESES}

Los autores declaran no tener conflicto de intereses. 


\section{CONTRIBUCIÓN POR AUTOR}

J.C.C-S y C.P. planearon, diseñaron y coordinaron la investigación. J.C.C-S. analizó los datos, realizó las tablas y redactó el manuscrito; C.P. supervisó la investigación, realizó comentarios y correcciones a los borradores anteriores; Los dos autores contribuyeron en igual medida a la elaboración del manuscrito.

\section{REFERENCIAS}

Adarve, J. B., Torres, A. M., Home, J., Vargas, J. A., Rivera, K., Duque, O. L. y González, A. M. (2010). Estructura y riqueza florística del Parque Natural Regional el Vínculo-Buga, Colombia. Cespedesia, 3g2(90/91), 23-38.

Akaike, H. (1974). A new look at the statistical model identification. En Selected Papers of Hirotugu Akaike (pp. 215-222). Nueva York: Springer.

https://doi.org/10.1007/978-1-4612-1694-0_16

Baskin, J. M. y Baskin, C. C. (2014). Ecology, Biogeography and Evolution of Dormancy and Germination. Academic Press.

Bedoya-Patiño, J. G., Estévez-Varón, J. V. y Castaño-ViIla, G. J. (2010). Banco de semillas del suelo y su papel en la recuperación de los bosques tropicales. Boletín Científico. Centro de Museos. Museo de Historia Natural, 14(2), 77-91.

Bhadouria, R., Singh, R., Srivastava, P. y Raghubanshi-Singh, A. (2016). Understanding the ecology of tree-seedling growth in dry tropical environment: a management prespective. Energy. Ecology and the Environment, 1(5), 296-309.

https://doi.org/10.1007/s40974-016-0038-3

Bolker, B. M. (2009). Ecological models and data in R. Princeton University Press.

Bond, W. R. y Parr, C. L. (2010). Beyond the forest edge: ecology, diversity and conservation of the grassy biomes. Biological Conservation, 143, 2395-2404.

https://doi.org/10.1016/j.biocon.2009.12.012

Bond, W. J., Honig, M. y Maze, K. E. (1999). Seed size and seedling emergence: an allometric relationship and some ecological implications. Oecologia, 120(1), 132-136.

https://doi.org/10.1007/s004420050841

Burnham, K. P. y Anderson, D. R. (2003). Model selection and multimodel inference: a practical information-theoretic approach. Springer Science \& Business Media.

Cardenas-Salgado., J. (2018). Efectos de altas temperaturas que simulan incendios sobre la germinación de semillas de un bosque seco tropical (trabajo de grado). Departamento de Biología, Facultad de Ciencias, Universidad Icesi, Cali, Colombia.

Carrington, M. E. (2010). Effects of soil temperature during fire on seed survival in Florida Sand Pine Scrub. International Journal of Forestry Research.

https://doi.org/10.1155/2010/402346

Cordell, S. y Sandquist, D. R. (2008). The impact of an invasive African bunchgrass (Pennisetum setaceum) on water availability and productivity of canopy trees within a tropical dry forest in Hawaii. Functional Ecology, 22(6), 1008-1017.

https://doi.org/10.1111/j.1365-2435.2008.01471.x

D'Antonio, C. M. y Vitousek, P. M. (1992). Biological invasions by exotic grasses, the grass/fire cycle, and global change. Annual Review of Ecology and Systematics, 23(1), 63-87.

https://doi.org/10.1146/annurev.ecolsys.23.1.63

Dokrak, M., Utis K., Hiroshi y Nakashizuka, T. (2004). Effects of drought and fire on seedling survival and growth under contrasting light conditions in a seasonal tropical forest. Journal of Vegetation Science, 16, 691-700.

https://doi.org/10.1111/j.1654-1103.2004.tb02311.x

Dryflor, B. R., Delgado-Salinas, A., Dexter, G. K., Linares-Palomino, R., Oliveira-Filho, A., Prado, D. y Weintritt, J. (2016). Plan diversity patterns in Neotropical dry forests and their conservation implications. Science, 353(6306), 1383-1387.

Erazo, A, L. (2016). Composición del banco de semillas germinable en un fragmento de bosque seco en el municipio de Patía, departamento del Cauca (proyecto de grado). Universidad del Cauca, Facultad de Ciencias Naturales, Departamento de Biología, Popayán, Cauca, Colombia. 
García, H., Corzo, G., Isaacs, P. y Etter, A. (2014). Distribución y estado actual de los remanentes del bioma de bosque seco tropical en Colombia: Insumos para su gestión. En C. Pizano y H. García (eds.), El bosque seco tropical en Colombia (cap. 8). Bogotá: Instituto de Investigación en Recursos Biológicos Alexander von Humboldt.

Goldammer, J. G. (ed.) (1993). Tropical Forests in Transition. Ecology of Natural and Anthropogenic Disturbance Processes. Basel, Switzerland: Birhauser-Verlag.

https://doi.org/10.1007/978-3-0348-7256-0_1

Gómez-González, S., Torres-Díaz, C., Bustos-Schindler, C. y Gianoli, E. (2011). Anthropogenic fire drives the evolution of seed traits. Proceedings of the National Academy of Sciences, 108(46), 18743-18747.

https://doi.org/10.1073/pnas.1108863108

Gómez-González, S., Paula, S., Cavieres, L. A. y Pausas, J. G. (2017). Postfire responses of the woody flora of Central Chile: Insights from a germination experiment. PloS one, 12(7), e0180661.

https://doi.org/10.1371/journal.pone.0180661

González-M, Pizano, C., R., Aguilar, J., Aguirre J., Barbosa A., Castaño-Naranjos, A., Duque A., Franke R., Galindo R., Idarraga, A., Jurado, R.D., López, R., Nieto J., Norden N., Pérez, K., Phillips J., Repizo A., Rodríguez G., Salgado-Negret B., Torres A. B. y García H. (2017). Monitoreo de la vegetación en los bosques secos de Colombia. En L. A. Moreno, G. I., Andrade y L. F. Ruiz-Contreras (eds.), Biodiversidad 2016. Estado y tendencias de la biodiversidad continental de Colombia. Bogotá, D.C., Colombia: Instituto Alexander von Humboldt.

González-M, R., García, H., Isaacs, P., Cuadros, H., López-Camacho, R., Rodríguez N., Pérez, K., Mijares, F., Castaño-Naranjo, A., Jurado, R., Idárraga-Piedrahíta, A., Rojas, A., Vergara, H. y Pizano, C. (2018). Disentangling the environmental heterogeneity, floristic distinctiveness and current threats of tropical dry forests in Colombia. Environmental Research Letters, 13(045007).

https://doi.org/10.1088/1748-9326/aaad74
Hanley, M., Unna, J. y Darvill, B. (2003). Seed size and germination response: a relationship for fire-following plant species exposed to thermal shock. Oecologia, 134(1), 18-22.

https://doi.org/10.1007/s00442-002-1094-2

Henao, N., Torres, A. M., Tafur, J. C. y Guevara, L. (2018) ¿Existe un efecto de borde sobre la estructura vegetal y potencial de regeneración en fragmentos de bosque seco tropical? Biota Colombiana, 19(1), 3-20.

https://doi.org/10.21068/c2018v19n01a01

Holdridge, L. R. (1967). Life zone ecology. San José, Costa Rica: Tropical Science Center.

Janzen, D. H. (1988). Tropical dry forests: the most endangered major tropical ecosystems. En E. O. WiIson (ed.), Biodiversity (pp. 130-136). Washington, D.C: National Academy Press.

Kennard, D. K. y Gholz, H. L. (2001). Effects of high-and low-intensity fires on soil properties and plant growth in a Bolivian dry forest. Plant and Soil, 234(1), 119-129.

https://doi.org/10.1023/a:1010507414994

Khurana, E. y Singh, J. (2001). Ecology of seed and seedling growth for conservation and restoration of tropical dry forest: A review. Environmental Conservation, 28(1), 39-52.

https://doi.org/10.1017/s0376892901000042

Labbé, D. L. (2013). Efecto del fuego sobre la viabilidad y germinación de semillas en ecosistemas Patagónicos (tesis de grado). Ingeniería en Conservación de Recursos Naturales, Universidad Austral de Chile, Valdivia, Chile.

Middleton, B. A., Sanchez-Rojas, E., Suedmeyer, B. y Michels, A. (1997). Fire in a tropical dry forest of Central America: a natural part of the disturbance regime? Biotropica, 29(4), 515-517.

https://doi.org/10.1111/j.1744-7429.1997.tb00045.x

Minitab, Inc. (2018). Minitab Statiscal Software. USA. Minitab, Inc.

https://doi.org/10.1002/9781119975328.advert

Monmany, A. C., Gould, W. A., Andrade-Núñez, M. J., González, G. y Quiñones, M. (2017). Characterizing predictability of fire occurrence in tropical forests and grasslands: the case of Puerto Rico. En S. 
Chakravarty y G. Shulka (eds.), Forest Ecology and Conservation (pp 77-95). InTechOPen.

https://doi.org/10.5772/67667

Morales G. A. (2016). Plan de manejo y conservación del Caracolì (Anacardium excelsum) en la jurisdicción CAR. Bogotá: CAR.

Norden, N., Daws, M. I., Antoine, C., Gonzalez, M. A., Garwood, N. C. y Chave, J. (2009). The relationship between seed mass and mean time to germination for 1037 tree species across five tropical forests. Functional Ecology, 23(1), 203-210.

https://doi.org/10.1111/j.1365-2435.2008.01477.x

Otterstrom, S. M., Schwartz, M. W. y Velázquez-Rocha, I. (2006). Responses to fire in selected tropical dry forest trees. Biotropica, 38(5), 592-598.

https://doi.org/10.1111/j.1744-7429.2006.00188.x

Pizano, C., González-M, R., López, R., Jurado, R.D., Cuadros, H., Castaño-Naranjos, A., Rojas, A., Pérez, K., Vergara-Varela, H., Idarraga, A. y García, H. (2016). El bosque seco tropical en Colombia: distribución y estado de conservación. En M. F. Gómez, L. A. Moreno, G. I. Andrade y C. Rueda (eds.), Biodiversidad 2015. Estado y tendencias de la biodiversidad continental de Colombia. Bogotá: Instituto Alexander von Humboldt.

Rodríguez-B, S, Corzo, G., García, H., Córdoba, D., Issacs, P. y Etter, A. (2016). Estrategias para la gestión integral de la biodiversidad. En M. F. Gómez, L. A. Moreno, G. I. Andrade y C. Rueda (eds.), Biodiversidad 2015. Estado y tendencias de la biodiversidad continental de Colombia. Bogotá: Instituto Alexander von Humboldt.

Romero-Saritama, J. M. y Pérez-Ruíz, C. (2016). Seed morphological traits and their implication in the ex situ conservation of woody species in Tumbesian dry forests. Ecosistemas, 25(2), 59-65.

https://doi.org/10.7818/ecos.2016.25-2.07
Torres, A. M., Adarve, J. B., Cárdenas, M., Vargas, J. A., Londoño, V., Rivera, K., ... y González, Á. M. (2012). Dinámica sucesional de un fragmento de bosque seco tropical del Valle del Cauca, Colombia. Biota Colombiana, 13(2).

Vargas, W. (2012). Los bosques secos del Valle del Cauca, Colombia: una aproximación a su flora actual. Biota Colombiana, 13(2).

Veldman, J. W., Mostacedo B., Peña-Claros M. y Putz F. E. (2009). Selective logging and fire as driver of alien grass invasion in a Bolivian tropical fry forest. Forest Ecology and Management, 258, 1643-1649.

https://doi.org/10.1016/j.foreco.2009.07.024

Veldman, J. W. y Putz F. E. (2010). Long-distance dispersal of invasive grasses by logging vehicles in a tropical dry forest. Biotropica, 42(6), 697-703.

https://doi.org/10.1111/j.1744-7429.2010.00647.x

Veldman, J. W. y Putz F. E. (2011). Grass-dominated vegetation, not species-diverse natural savanna, replaces degraded tropical forests on the southern edge of the Amazon Basin. Biological Conservation, 144, 1643-1649.

https://doi.org/10.1016/j.biocon.2011.01.011

Vieira, D. L. y Scariot, A. (2006). Principles of natural regeneration of tropical dry forests for restoration. Restoration Ecology, 14(1), 11-20.

https://doi.org/10.1111/j.1526-100x.2006.00100.x

Wanthongchai, K., Goldammer, J. G. y Bauhus, J. (2011). Effects of fire frequency on prescribed fire behaviour and soil temperatures in dry dipterocarp forests. International Journal of Wildland Fire, 20(1), 35-45.

https://doi.org/10.1071/wf08098

Wickham, H. (2005). Ggplot2. R package version 1.14.4. 
Anexo 1. Evaluación del criterio de información de Akaike para los distintos modelos estadísticos. El modelo que mejor se ajusta a los datos está resaltado en negrilla.

\begin{tabular}{|c|c|c|}
\hline Enterolobium cyclocarpum & Guazuma ulmifolia & Sapindus saponaria \\
\hline $\begin{array}{c}\text { AIC dAIC df } \\
\text { m4_ec 14.4 } 0.03 \\
\text { m2_ec 15.3 } 0.84 \\
\text { mnull_ec 16.8 2.4 1 } \\
\text { m1_ec 17.3 } 2.85 \\
\text { m3_ec 19.1 } 4.73 \\
\end{array}$ & $\begin{array}{c}\text { AIC dAIC df } \\
\text { m4_gu 14.4 } 0.03 \\
\text { m2_gu 15.3 } 0.84 \\
\text { mnull_gu 16.8 2.4 1 } \\
\text { m1_gu 17.3 2.8 } 5 \\
\text { m3_gu 19.1 4.7 3 } \\
\end{array}$ & $\begin{array}{c}\text { AIC dAIC df } \\
\text { m2_ss 14.9 } 0.04 \\
\text { m4_ss 15.1 } 0.23 \\
\text { m1_ss 16.9 } 2.05 \\
\text { mnull_ss 18.4 } 3.51 \\
\text { m3_ss 20.3 } 5.4 \\
\end{array}$ \\
\hline Pithecellobium dulce & Ceiba pentandra & Senna spectabilis \\
\hline $\begin{array}{c}\text { AIC dAIC df } \\
\text { m4_pd 12.7 0.0 3 } \\
\text { m2_pd 14.7 } 2.04 \\
\text { m1_pd 16.7 } 4.05 \\
\text { m3_pd 107.6 } 94.83 \\
\text { mnull_pd 113.6 } 100.81\end{array}$ & $\begin{array}{c}\text { AIC dAIC df } \\
\text { m4_cp 17.9 0.0 3 } \\
\text { m2_cp 18.2 } 0.34 \\
\text { m1_cp 20.2 2.3 } 5 \\
\text { mnull_cp 60.6 42.6 } 1 \\
\text { m3_cp 60.9 42.93 }\end{array}$ & $\begin{array}{c}\text { AIC dAIC df } \\
\text { m4_sp 16.6 0.0 3 } \\
\text { m2_sp 17.0 0.4 } 4 \\
\text { m1_sp 19.0 2.4 } 5 \\
\text { m3_sp 25.5 } 8.93 \\
\text { mnull_sp 28.4 } 11.71\end{array}$ \\
\hline
\end{tabular}

Erythryna poeppigiana

\section{Anacardium excelsum}

\begin{tabular}{|c|c|}
\hline $\begin{array}{c}\text { AIC dAIC df } \\
\mathrm{m} 4 \text { ep } 13.80 .03\end{array}$ & $\begin{array}{c}\text { AIC dAIC df } \\
\text { mnull ae } 7.20 .01\end{array}$ \\
\hline $\mathrm{m} 2$ ep 15.82 .04 & $\mathrm{~m} 3$ ae 7.90 .73 \\
\hline mnull_ep 17.73 .81 & m4_ae 7.90 .73 \\
\hline m1_ep 17.84 .05 & m2_ae 9.92 .74 \\
\hline m3_ep 19.65.8 3 & m1_ae 11.94 .75 \\
\hline
\end{tabular}

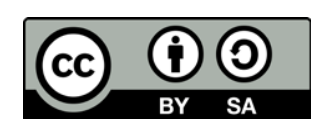

